\title{
Ein niedriger PSA-Wert kann auch ein schlechtes Zeichen sein
}

\begin{abstract}
Hoher Gleason-Score, aber PSA-Wert unter $2,5 \mathrm{ng} / \mathrm{ml}$ : Das ist alles andere als eine günstige Konstellation, wie eine Studie ergab. Brandon A. Mahal und Kollegen nahmen sich die Angaben zu knapp einer viertel Million Patienten aus dem SEER(Surveillance, Epidemiology, and End Results)-Programm vor. Sie betrafen alle Männer mit klinischen T1-4N0M0Karzinomen. Median lief die Nachbeobachtung 38 Monate. Spezielles Augenmerk galt Patienten mit einem Gleason-Score von 8-10. Hier zeigte sich eine U-förmig verlaufende Sterblichkeitskurve bezogen auf die PSA-Konzentration. Als Referenzwert diente ein PSA-Wert von 4,1-10,0 ng/ ml; die kumulative 5-Jahres-Mortalität lag hier rechnerisch bei rund $5 \%$.
\end{abstract}

Erwartungsgemäß war das Risiko, an Prostatakrebs zu sterben, am höchsten (mehr als 3-mal so hoch), wenn der PSA-
Wert über 40,0 ng/ml lag (Hazard Ratio $3,23)$. Bei einem PSA zwischen 20,1 und $40,0 \mathrm{ng} / \mathrm{ml}$ war die Sterblichkeit 2,08mal, bei einem Wert von 10,1 bis 20,0 ng/ $\mathrm{ml}$ 1,60-mal höher als bei Männern mit dem Referenz-PSA-Wert.

Erhöht war die prostatakrebsspezifische Mortalität aber auch, wenn bei einem Gleason-Score von 8-10 der PSAWert höchstens $2,5 \mathrm{ng} / \mathrm{ml}$ betrug; das spezifische Sterberisiko war hier um den Faktor 2,15 gesteigert und führte zu einer kumulativen prostatakrebsspezifischen 5-Jahres-Mortalität von rund $10 \%$.

Der Verlauf der Kurve, welche die Mortalitätsraten in Abhängigkeit vom PSAWert bei einem Gleason-Score von 8-10 widerspiegelt, unterscheidet sich somit von jener, die sich bei Gleason-Scores $\leq 7$ ergibt. Hier gibt sich ein Risikoverlauf, der bis zu einem Wert von $4,1 \mathrm{ng} / \mathrm{ml}$

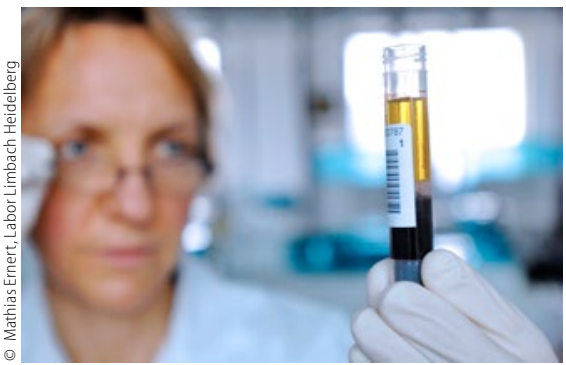

Bei Prostatakarzinomen mit hohem Gleason-Score ist ein niedriger PSA-Wert ein Grund zur Beunruhigung.

horizontal und indifferent verläuft und erst danach in höhere Werte ansteigt.

Die relativ hohe Sterblichkeit bei hohem Gleason-Score und niedrigem PSAWert legt für Mahal und Mitarbeiter eine tumorpathologische Vermutung nahe. Niedrige PSA-Konzentrationen seien hier womöglich ein Zeichen für aggressiv wachsende, extrem schlecht differenzierte oder anaplastische Karzinome, die wenig PSA produzieren.

Robert Bublak

Mahal BA et al. Association of Very Low ProstateSpecific Antigen Levels With Increased CancerSpecific Death in Men With High-Grade Prostate Cancer. Cancer. 2015 Sep 15. [Epub ahead of print].

\section{Komorbidität beeinflusst Gesamtüberleben}

Das Gesamtüberleben eignet sich möglicherweise nicht dafür, den Erfolg spezifischer Behandlungen, etwa mit Tyrosinkinaseinhibitoren, bei Patienten mit chronischer myeloischer Leukämie (CML) zu messen, so das Ergebnis einer aktuellen Studie, in der Imatinib in der Erstlinie in verschiedenen Dosierungen und Kombinationen zum Einsatz kam. In dieser Studie wurden auch Komorbiditäten als möglicher Einflussfaktor untersucht.

Bei 1.519 CML-Patienten wurden anhand des Charlson-Comorbidity-Index (CCI) 511 Komorbiditäten registriert. Der CCI-Index berücksichtigt nicht nur das Auftreten von Komorbiditäten, sondern auch deren Schwere. Bei 863 Patienten kam ein höheres Lebensalter als zusätzlicher Risikofaktor hinzu.

Hinsichtlich der Komorbiditäten ergab sich folgendes Bild: CCI 2: 589 Patienten, CCI 3 oder 4: 599 Patienten, CCI 5 oder 6: 229 Patienten, CCI $\geq 7: 102$ Patienten. Hö- here CCI-Werte waren mit einem signifikant geringeren Gesamtüberleben assoziiert. Die 8-Jahres-Gesamtüberlebensrate betrug für den CCI-Score $293,6 \%, 89,4 \%$ für die CCI-Scores 3-4, 77,6\% für die CCI-Scores 5-6 und 46,4\%für Scores $\geq 7$.

In der Multivarianzanalyse erwies sich der CCI als der stärkste Prädiktor für das Gesamtüberleben, auch nach Bereinigung altersbedingter Komponenten. Jedoch profitierten Patienten mit multiplen Komorbiditäten von der Imatinibtherapie: Es gab keine negativen Effekte auf die Remissionsraten und die Progression der Krankheit; eine gute molekulare Remission und eine zytogenetische Remission konnten ebenso erreicht werden wie bei Patienten ohne Komorbiditäten.

Kathrin von Kieseritzky

Saußele S et al. Impact of comorbidities on overall survival in patients with chronic myeloid leukemia: results of the randomized CML Study IV. Blood. 2015;126(1):42-9.

\section{kurz notiert}

Forschungsstandort Deutschland

Reparaturenzym schützt vor Krebs +++ Mainzer Forscher zeigten im Tiermodell, dass bei Fehlen des DNA-Reparaturenzyms O6-Methylguanin-DNA-Methyltransferase (MGMT) bereits eine niedrige Dosis eines Karzinogens zu Darmkrebs führen kann. Im Gegensatz dazu bildeten sich keine Tumoren, wenn ein normales DNA-Reparaturenzym oder ein anderer Reparaturdefekt vorlag. Diese Erkenntnis stellt das Konzept der Schwellendosen von Karzinogenen infrage, was Konsequenzen für die Bewertung krebserregender Stoffe haben könnte [Fahrer J et al. Carcinogenesis. 2015;36(10):1235-44].

Hoffnung auf epigenetische Therapie +++ Zellen des Pankreaskarzinoms bilden besonders viel BRD4, ein Protein, das den epigenetischen Code von Zellen reguliert. Wissenschaftler aus München und Stanford, CA/USA, konnten nun im Tiermodell zeigen, dass das gegen BRD4 gerichtete "small molecule" JQ1, das Wachstum eines Prostatakarzinoms verringert. Am effektivsten war die Therapie, wenn sie mit einer weiteren epigenetisch wirkenden Substanz, SAHA, einem Inhibitor der Histondeacetylase, kombiniert wurde. Dadurch konnte das Überleben der Tiere deutlich verlängert werden [Mazur PK et al. Nat Med. 2015;21(10):1163-71]. 